\title{
reviscafuences
}

ISSN: 1575.7072 | 8.15SN: 2172.7775

Páginas: 244-253

Recibido: 2021-02-23

Revisado: 2021-03-02

Aceptado: 2021-04-19

Preprint: 2021-05-10

Publicación Final: 2021-05-15

www.revistascientificas.us.es/index.php/fuentes/index

DOI: https://doi.org/10.12795/revistafuentes.2021.15375

\section{La democracia en los centros educativos españoles: concepciones de docentes y estudiantes}

\author{
Democracy in spanish schools: conceptions of teachers and students
}

\author{
(iD) Guillermina Belavi \\ Universidad Autónoma de Madrid (España) \\ (iD) Carlos Flores-Sánchez \\ Universidad Autónoma de Madrid (España) \\ (iD) Claudia Guiral \\ Universidad Autónoma de Madrid (España) \\ (iD) Yasin Türk \\ Universidad Autónoma de Madrid (España)
}

\begin{abstract}
Resumen
Las concepciones de docentes y estudiantes sobre la democracia influyen en las actitudes, las expectativas y las posibilidades de esta forma de gobierno, por ello resulta fundamental conocerlas. El objetivo de esta investigación es describir las concepciones de democracia en el centro educativo que tienen docentes y estudiantes en España. Para ello se realizó una investigación fenomenográfica cuyos participantes fueron 15 profesores y 15 alumnos de centros educativos españoles. Los resultados sugieren tres concepciones sobre la democracia en el centro escolar, aunque con ligeras diferencias entre ambos grupos. En primer lugar, una concepción elitista que reduce la democracia a la libre competencia y elección de centro por parte de las familias, cuyos discursos jerarquizados resultan afines a la filosofía neoliberal. Una representativa que enfatiza el voto como mecanismo de decisión. Las principales diferencias entre docentes y estudiantes se encuentran bajo esta concepción, ya que mientras el profesorado tiende a desestimar el poder real del estudiantado, el alumnado llega a reclamar injerencia en decisiones trascendentales del centro. Finalmente, una concepción participativa que se caracteriza por la horizontalidad y la toma de decisiones consensuada. Promover esta concepción participativa resulta fundamental para fomentar el pensamiento crítico y la intervención de todas las personas en los asuntos comunes, avanzando así hacia sociedades más justas y equitativas.

\section{Abstract}

Teachers' and students' conceptions of democracy influence the attitudes, expectations, and possibilities of this form of government; therefore, it is essential to know them. This research aims to describe the conceptions of democracy in the school held by teachers and secondary school students in Spain. With that purpose, a phenomenographic study was carried out. Participants were 15 teachers and 15 students attending schools in Spain. Results indicate that there are three conceptions of democracy in school, although with differences between groups. First, an elitist conception that reduces democracy to free competition and school choice. Discourses held under this conception are related to the neoliberal philosophy. A representative conception that emphasizes voting as a decision mechanism. The main differences between teachers and students are found here. While teachers tend to underestimate the real power of students, the second group claims interference in the transcendental school decisions. Finally, a participatory conception characterized by horizontality and consensus decision-making. To promote a participatory conception of democracy is essential to encourage critical thinking and collective agency in common issues, thus advancing towards more just and equitable societies.
\end{abstract}

\section{Palabras clave}

concepciones, democracia, educación, escuela, ciudadanía, participación, representación, elitismo. 


\section{Keywords}

conceptions, democracy, education, school, citizenship, participation, representation, elitism.

\section{Introducción}

La forma en la que se comprende un fenómeno condiciona la manera de actuar, nuestras expectativas y el valor que se le otorga. Las concepciones sobre la democracia, por lo tanto, influyen en las actitudes, las expectativas e incluso el apoyo de las personas a esta forma de gobierno (Bengtsson y Christensen, 2016; Ceka y Magalhães, 2020; Landwehr y Steiner, 2017). En educación esto también es cierto (Zyngier, 2016), por ello el presente artículo se interesa por las concepciones de docentes y estudiantes sobre la democracia en el centro educativo.

La investigación educativa aborda el tema desde distintos puntos de vista. Existen estudios que recogen las opiniones del profesorado (Ginocchio et al., 2015; Vidal et al., 2019; Zyngier, 2011), de administradores educativos y miembros de la sociedad (Long, 2018; Carr et al., 2018), de profesorado en formación (Carr, 2008; Carr et al., 2018; Hung y Perez, 2020; Zyngier, 2011; Zyngier, 2016; Zyngier et al., 2015) y de estudiantes de educación primaria o secundaria (Arensmeier, 2010; Kus y Çetin, 2014; Mathé, 2016; Quaranta, 2020).

La investigación Democracia, alfabetización política y educación transformadora (Carr et al., 2018; Zyngier, 2013) es referencia obligada en el tema, ya que busca comprender cómo es conceptualizada y experimentada la democracia en y a través de la educación. A partir de trabajos de Barber $(1984,1989)$ y de Gandin y Apple (2002), considera la democracia desde un «espectro grueso-delgado» (Thick-Thin Spectrum) (Carr, 2008; Carr y Thésée, 2017; Carr et al., 2018) que caracteriza la democracia «en términos de una democracia representativa versus una participativa, enfatizando los procesos electorales la primera (delgada) mientras que la segunda se enfoca en la participación crítica y la justicia social (gruesa)» (Carr, 2008, p. 147). La investigación sugiere que las concepciones que priman en docentes y estudiantes de magisterio son, en su mayoría, delgadas: enfatizan elecciones, gobierno, estructuras y procesos políticos hegemónicos. Apenas existe mención a enfoques relacionados con un espectro grueso de la democracia y casi nunca se establece un vínculo significativo entre la democracia y la educación (Carr et al., 2018; Zyngier et al., 2015).

Las investigaciones que indagan las concepciones de democracia del estudiantado (Arensmeier, 2010; Kuş y Çetin, 2014; Mathé, 2016, 2019) indican que predominan visiones asociadas a una democracia representativa liberal, donde los procedimientos y las instituciones tienen mayor peso en la definición que los valores y la participación. Entre ellas destaca la de Mathé $(2016,2019)$, que analiza las concepciones de democracia del alumnado noruego de 16 años. Los resultados señalan que predomina una comprensión liberal de la democracia que resalta el voto como la actividad política principal, también denominada democracia electoral (Munck, 2016).

Por su parte, Arensmeier (2010) estudia la concepción de democracia en estudiantes suecos de educación obligatoria (14/15 años) y en el último año de educación secundaria (18/19 años). El conjunto de participantes de este estudio respalda la democracia, pero la consideran un asunto problemático. Expresan dudas sobre la capacidad humana para ejercer un autogobierno y se muestran escépticos respecto al poder real de la ciudadanía. En definitiva, predomina una concepción de la democracia electoral o de élite.

Otro grupo de investigaciones estudia las concepciones que tienen docentes sobre ciudadanía democrática (Biseth, 2011; Cohen, 2016a; Hahn, 2015; Hung y Perez, 2020; Saada, 2020; Sim et al., 2017). Trabajan con perfiles específicos, como profesorado de educación cívica, de modo que hallan ideas más complejas y profundas sobre la democracia y llegan a una misma conclusión: las concepciones de ciudadanía de los docentes influyen en la planificación y el desarrollo de sus clases.

Esta idea nos lleva a otro grupo de estudios que tratan explícitamente la relación entre democracia (o ciudadanía democrática) y educación (Cohen, 2016b; Edling y Liljestrand, 2018; Ginocchio et al., 2015; Westheimer y Kahne, 2004). Dentro de este grupo, destacamos estudios realizados en el ámbito español. Se trata de investigaciones que indagan sobre las concepciones de democracia o de participación democrática en docentes y su relación con las escuelas (Ferreras-Listán et al., 2018; Sainz-Linares et al., 2018).

Sainz-Linares et al. (2018) analizan los discursos del profesorado acerca de la participación del estudiantado en las escuelas. Se basan en la premisa de que los diferentes discursos remiten a modelos distintos de democracia que inspiran, a su vez, prácticas escolares diversas. Según los resultados, las concepciones se ubican dentro de un amplio espectro cuyos extremos son, por un lado, concepciones que asocian la participación con características individuales de las personas y, por el otro, discursos que enfatizan una perspectiva sociopolítica y refieren al derecho de intervención en la toma de decisiones y en la construcción del bien común. Predominan las concepciones ubicadas en el primer rango.

La revisión de estos estudios permite conocer el estado de la cuestión y sugiere, al mismo tiempo, una pregunta: ¿qué concepciones existen en el contexto español sobre la democracia en el centro educativo? El objetivo de 
esta investigación es, por tanto, describir las concepciones de democracia en el centro educativo que tienen docentes y estudiantes de educación obligatoria en el ámbito español. Este objetivo contribuye a la literatura existente en tanto busca analizar las concepciones de dos grupos protagonistas de la educación (docentes y estudiantes) en lo que refiere a la democracia en el centro educativo específicamente (y no a la democracia como concepto genérico).

\section{Metodología}

Para cumplir con el objetivo se realizó una investigación fenomenográfica, pues la fenomenomenografía es el método idóneo para comprender el modo en el que las personas interpretan los aspectos de su mundo, es decir, sus concepciones (Marton, 1986). En efecto, permite comprender las diferentes formas en que los individuos experimentan y dotan de significado a un fenómeno determinado (Orgill, 2012; Marton y Booth, 1997). Las concepciones son singulares para cada individuo, no obstante, pueden encontrarse similitudes entre ellas y así agruparlas en categorías conceptuales (Marton, 1981). La fenomenografía, por lo tanto, permite reducir y comparar los significados que los sujetos atribuyen a la democracia en el centro escolar.

La selección de participantes es un aspecto esencial dentro del enfoque fenomenográfico, pues debe garantizarse un abanico amplio de visiones en torno a un mismo concepto (Durden, 2018; Marton y Booth, 1997). Para ello, en este estudio se seleccionaron como participantes a 15 docentes y 15 estudiantes de centros educativos situados en la Comunidad de Madrid y en Andalucía atendiendo a los siguientes criterios:

- Titularidad del centro: diferenciando entre centros públicos y centros privados.

- Nivel educativo: se seleccionaron profesores de educación primaria y secundaria, y estudiantes de educación secundaria al considerarse que poseían una concepción más desarrollada del fenómeno que el estudiantado de primaria.

- Contexto socioeconómico: se diferenció entre centros escolares situados en contextos favorables y desafiantes. Esta categorización se hizo de acuerdo con el nivel socioeconómico y cultural del barrio en el que se ubica el centro.

En el siguiente cuadro se presenta la distribución de los participantes en relación con los criterios señalados:

Tabla 1

Distribución de la muestra

\begin{tabular}{|c|c|c|c|c|c|c|}
\hline & & Educaci & Primaria & $\begin{array}{l}\text { Educaci } \\
\text { FP }\end{array}$ & undaria, Bachillerato y & Total \\
\hline & & Público & Privado & Público & Privado & \\
\hline & Favorable & 0 & 0 & 2 & 5 & 7 \\
\hline Estudiantes & Desafiante & 0 & 0 & 5 & 3 & 8 \\
\hline & Favorable & 3 & 3 & 1 & 2 & 9 \\
\hline Docentes & Desafiante & 2 & 0 & 4 & 0 & 6 \\
\hline & TOTAL & 5 & 3 & 12 & 10 & 30 \\
\hline
\end{tabular}

La entrevista semi-estructurada es el método más habitual en la recogida de información dentro de la investigación fenomenográfica, pues permite analizar en profundidad las distintas formas en las personas experimentan o perciben el fenómeno a estudiar (González-Ugalde, 2014; Murillo y Hidalgo, 2018). Para ello, se procura que la persona entrevistada reflexione sobre sus propias experiencias en torno a un fenómeno en particular (González-Ugalde, 2014). En este caso, el guion de la entrevista consta de dos preguntas generadoras que permiten profundizar en las concepciones del o la participante: «¿Qué es para ti la democracia?» $\mathbf{y}$ « ¿Qué es para ti la democracia en la escuela?».

Una vez transcritas las entrevistas, se realizó el análisis de los datos siguiendo el proceso propuesto por Marton (1986) que puede describirse en tres pasos:

En primer lugar, se seleccionaron las citas más relevantes para el objetivo de investigación, haciendo el esfuerzo de apartar las ideas preconcebidas sobre el fenómeno y así permitir la aparición de nuevos significados (Akerlind, 2008). De este modo, el análisis inicial no incluía categorías previas.

El segundo paso consistió en establecer pre-categorías en base a un trabajo interpretativo y dialéctico de agrupación, reagrupación y comparación en torno a las similitudes y las diferencias de los significados extraídos de las citas seleccionadas. En este punto resultó crucial el análisis basado en las propias palabras de las personas entrevistadas para definir la categoría de significado a la que pertenecen. La selección de 
los elementos más significativos para la definición de las categorías se hizo de acuerdo a los indicadores propuestos por Sjöström y Dahlgren (2002), a saber, la frecuencia con la que aparece una idea, la posición que ocupa y el énfasis que se le da. Este segundo paso se realizó de forma reiterativa, por lo que implicó numerosas revisitas a los datos por parte de los cuatro investigadores para confirmar la validez de las categorías que emergían de las entrevistas y asegurar credibilidad del proceso interpretativo de acuerdo con los criterios de calidad propuestos por Sin (2010).

Posteriormente, se afinaron las pre-categorías para asegurar la relación estructural entre los significados encontrados y se les atribuyeron criterios explícitos para definirlas. De este modo, se establecieron tres categorías de descripción que representan formas cualitativamente diferentes de entender la democracia en la escuela, es decir, tres concepciones que pueden ser ejemplificadas mediante las citas seleccionadas y que, en su conjunto, representan todos los significados potenciales del fenómeno estudiado.

Para asegurar el rigor del proceso de análisis se usó el programa informático Atlas.ti.

\section{Resultados}

Las respuestas de docentes y estudiantes acerca de qué significa la democracia en la escuela presentan diferencias y matices que sugieren tres concepciones centrales: una elitista (tecnocrática), otra representativa y una que enfatiza la participación. A continuación, se describe qué implica cada una de estas concepciones y cuáles son las principales diferencias o matices que se perciben entre las ideas de docentes y estudiantes ante una misma concepción.

\subsection{Concepción elitista de la democracia}

Existe una concepción que asocia la democracia a un método de legitimación de quienes toman las decisiones, donde el papel de la ciudadanía en los asuntos de gobierno es muy limitado. Siguiendo la literatura académica, se llama «concepción elistista» a esta forma de comprender la democracia. Esta visión puede rastrearse en autores como Michels (2008), que asocia la democracia con un mero método político, o Schumpeter (1988), quien la relaciona con la libre competencia y la libertad de elección. Según estas ideas, en cualquier organización es inevitable la jerarquía y las posiciones de poder, que indefectiblemente serán ocupadas por un pequeño grupo de personas.

Esta concepción se encuentra presente a la hora de consultar a docentes y estudiantes sobre la democracia en la escuela, tal como lo refleja la siguiente cita del alumnado de Educación Secundaria:

... la democracia está cuando tú eliges el instituto. Cuando te metes en el instituto tú estás aceptando las reglas [...] Una vez estás dentro del colegio tú ya tienes que seguir las reglas, no puede haber democracia y decir pues yo ahora quiero cambiar esta regla...

(4_E_Sec_Pri_Fav)

La libertad de elección de centros es el verdadero momento democrático en educación y el mecanismo que legitima el funcionamiento de la escuela. Esta visión está relacionada con filosofías neoliberales, basadas en la «libre competencia» de los centros escolares por el cual se establece un ranking con los «mejores centros». Las políticas de libertad de elección de centro son, por lo tanto, fundamentales. Esta idea se refleja entre el profesorado: «De alguna manera, la libertad de los padres de elegir los centros y el tipo de educación que desean para sus hijos debe respetarse.» (13_D_Sec_Priv_Fav)

Una vez escogido el centro, se asume que las decisiones serán tomadas por las autoridades, no teniendo cabida el cuestionamiento o la autogestión. Una postura tecnocrática también subyace a esta concepción: puesto que la jerarquía es inevitable y solo unos pocos ocuparán los lugares de poder, es deseable que sean especialistas y técnicos los que pueden imponer su criterio sobre un determinado tema.

[...] el profesor es el técnico y el que tiene que decir «esto es lo que necesitáis. [...] ¿Tú crees que realmente algún niño haría algo si no hubiera profesores? [...] Tiene que haber por fuerza, alguien que dirija, mande y organice el proyecto.

(5_D_Sec_Pub_Des)

Bajo esta concepción, la función docente deriva de sus conocimientos y el estudiantado la requiere, por lo tanto, en una relación de dependencia. El siguiente testimonio docente refleja la idea de organización jerárquica que diferencia entre alumnado y docentes: «Yo creo que cada uno tiene su sitio y sus funciones, y en el momento 
que me extrapolo y creo que puedo llegar a otros territorios, [...] ahí es donde lamentablemente hay que poner un límite»(22_D_Pri_Pub_Fav).

Al igual que entre el profesorado, el estudiantado comparte esta visión jerárquica y técnica y rechaza su propia participación en el proceso de toma de decisiones de la escuela: «...cuando le das libertad [al alumnado] yo creo que nosotros nos subimos y ya creemos que tenemos el poder, pero para que estemos todos tranquilos, debe haber una jerarquía» (4_E_Sec_Pri_Fav).

Llama la atención la desconfianza hacia el comportamiento de la juventud y hacia lo que pueden lograr por sí mismos. Fundamentalmente cuando el propio estudiantado asume un rol pasivo de legitimación de las normas y admite su incapacidad para transformarlas:

No sé por qué se lleva uniforme pero algún motivo tendrá cuando lo ha puesto que se lleve. Entonces como yo no estoy capacitada como para saber estas cosas, ese tipo de cosas, ya no eso, sino a niveles más grandes, decisiones más grandes, no podría decidir.

(1_E_Sec_Pri_Fav)

Bajo esta concepción, la democracia queda supeditada a un medio de legitimación por el cual las familias, al escoger el centro, avalan la enseñanza que se brinda y confían el poder de decisión a las autoridades educativas. El alumnado no tiene espacio para participar en las decisiones.

Docentes y estudiantes comparten un mismo esquema verticalista de autoridad y existen pocos matices y diferencias entre las ideas de estos colectivos, a no ser que consideremos la distancia y los roles diametralmente opuestos que asume cada grupo, que pueden resumirse en un arquetipo: el profesorado tiene el conocimiento y la autoridad; mientras que el estudiantado carece del conocimiento y de la aptitud. Su rol, por lo tanto, es el de acatar las normas ya establecidas.

\subsection{Concepción representativa de la democracia}

Los resultados muestran otra concepción de democracia en las escuelas que puede definirse como representativa. Desde esta concepción, la democracia se vincula al voto como principal mecanismo de decisión, a las elecciones de representantes y a los órganos formales dentro del colegio. A diferencia de la concepción elitista, aquí toda la comunidad educativa participa en las decisiones del centro (incluido el estudiantado), aunque esto merece cierta aclaración.

La participación universal y directa se circunscribe a la acción de votar a representantes. A través del voto, los electores delegan su poder para que otros decidan en su nombre. Una vez escogidos los representantes, la participación en las decisiones del centro estará mediada por su figura. Así pues, se establece una división entre las personas con algún tipo de responsabilidad (quienes participan en la toma de decisiones) y el resto (quienes delegan su poder de decisión).

Dentro de esta concepción, la democracia en la escuela se relaciona con órganos representativos, especialmente con el consejo escolar, donde se toman las decisiones del centro. Las decisiones no se consideran un asunto técnico y restringido. Por el contrario, la legitimidad democrática se halla en el diálogo entre los distintos representantes, que permite a todas las voces formar parte del debate de los asuntos comunes. El siguiente testimonio docente sirve de ejemplo:

En los órganos donde estamos representados o en los diferentes niveles que hay en la escuela, (...) en la coordinación del tramo, o en la CCP, o en el claustro, o en los consejos escolares: que todas las voces estemos allí, estemos representadas y podamos decidir entre todos [...] en esos órganos colegiados podemos decidir ciertas cosas, que no vengan impuestas [...] desde la dirección.

$$
\text { (3_D_Pri_Pub_Des) }
$$

Dentro de esta concepción no todos los grupos tienen el mismo poder de influencia. El alumnado, aunque ahora puede opinar, no siempre tiene injerencia en las decisiones últimas, manteniendo así un papel subordinado. Esto se observa fundamentalmente en el testimonio de docentes:

Nosotros tenemos el consejo escolar [...] [donde] están representados todos los grupos de la comunidad educativa y ahí es donde se plantean problemas, posibles cosas y se aprueban y todo [...] En el colegio, el órgano de gobierno es el consejo, donde está totalmente reflejado ese espíritu democrático. [...] Y están representados, hasta te digo, los estudiantes, lo que pasa es que ellos solo tienen voz, no tienen voto.

(8_D_Pri_Priv_Fav) 
Algunos docentes valoran poder participar en las decisiones sin que éstas les vengan impuestas desde la dirección, pero consideran la participación del estudiantado una concesión y admiten su lugar secundario. De esta manera, la igualdad democrática no se realiza completamente, ni siquiera a través de la igualdad entre representantes.

La concepción representativa del estudiantado tiene puntos de encuentro y de desencuentro con respecto a los docentes. El núcleo de definición es el mismo: la democracia en la escuela significa elegir a representantes y participar, a través de su mediación, en las decisiones del centro. Incluso comparten la postura ambivalente en cuanto a la participación de quienes tradicionalmente tienen «menos autoridad», aunque adaptado a su grupo etario:

[En una escuela democrática] supongo que se debería tener un representante en cada aula, una especie de sindicato de alumnos, más movimiento por nuestra parte. [...] Quizá en secundaria sí y en primaria no tanto, $[\ldots]$ es que no le vas a decir a un niño de diez años $[\ldots]$ porque posiblemente lo que voten será lo que le convenga más a priori.

$$
\text { (6_E_Sec_Pub_Des) }
$$

Dentro de esta misma concepción, sin embargo, aparece otra perspectiva del estudiantado que destacamos como diferencia respecto al profesorado. Según esta visión, la democracia exige el poder de todos y todas de influir incluso en las decisiones más importantes y trascendentales para el centro, en aquellas que resultan estructurales a la hora de definir la educación que brindará el centro educativo. Así lo refleja la siguiente cita:

Una clase sí es democrática porque, por ejemplo, nosotros escogemos a nuestros delegados, o por ejemplo cuando escogemos a los alumnos en el consejo escolar [...], pero no es democrático del todo porque al director no le escogemos los alumnos. Entonces sería más democrático si le escogiesen los alumnos.

$$
\text { (5_E_Sec_Pri_Fav) }
$$

Como se observa, el esquema representativo se mantiene: no se propone una dirección comunitaria ni una participación (más o menos directa) en las decisiones de dirección, sin embargo, se defiende que el alumnado tenga el poder de escoger a la persona que estará a cargo de su centro educativo.

\subsection{Concepción participativa de la democracia}

Por último, encontramos una concepción de democracia que puede definirse como participativa y que defiende que toda la comunidad educativa participe en la toma de decisiones que les afectan. La comunidad educativa se entiende de una manera amplia, incluyendo a las familias y a la comunidad en general: «[Se trata de] proponer unos temas, debatirlos y decidirlos entre todos, incluyendo a familias también» (4_D_Pri_Pub_Des). Esta participación, por principio, tiene que ser igualitaria, de manera que se defiende la inclusión y la horizontalidad en las relaciones, tal como se refleja en la siguiente cita docente:

Pues para mí [la democracia en la escuela] es una forma de organización en la que no existen ni grados ni estatus y en el que todo el mundo estaría en el mismo nivel a la hora de organizar, de decidir, de tomar decisiones...de organizar el día a día en la escuela.

$$
\text { (16_D_Sec_Priv_Fav) }
$$

Esta horizontalidad rompe con las dicotomías de las concepciones anteriores, tanto la de alumnadoprofesorado como la de representantes-representados. En lugar de establecerse una relación vertical como en la concepción elitista, ahora se espera que todos participen en las decisiones del centro y del aula:

Democracia [significa] consensuar acuerdos. Una libre participación de todos los miembros para tomar decisiones de manera común. [En el aula] yo propongo un tema [...] y los niños son los que enfocan cómo lo vamos a trabajar. Yo hago siempre una lluvia de ideas en las que ellos exponen que es lo que les interesa, cuáles son sus motivaciones. A partir de esas motivaciones empezamos a trabajar.

$$
\text { (12_D_Pri_Pub_Fav) }
$$

Esta misma idea se observa entre el alumnado, quien también considera que resulta fundamental participar en la toma de decisiones del centro: 
Un instituto donde todos pudiéramos decidir. No que solo decidan los profesores por el hecho de ser profesores, que no tomen las decisiones por nosotros. [...] Obviamente los profesores saben lo que nos conviene y lo que deberíamos de dar, [...] pero a lo mejor la forma de dar los contenidos sí se nos puede preguntar. El profesor piensa que es mejor de una forma y nosotros de esa forma no nos enteramos, entonces poder proponer otra forma de hacerlo [...] y debatir sobre qué es mejor.

$$
\text { (4_E_Sec_Pub_Fav) }
$$

Como se mencionó antes, la dicotomía entre representantes y representados pierde peso bajo esta concepción, pues las normas del centro se deciden de forma común y a través de la participación directa. Cuando esta participación no existe, las consecuencias pueden afectar incluso al proceso educativo, como destaca esta cita docente:

Evidentemente en el instituto tiene que haber una serie de normas, [...] esas normas [...] tienen que ser construidas por todos [...]. Y esto no pasa actualmente. Y, de hecho, se nota que hay una falta de implicación de los alumnos. Si realmente este proceso democrático se diera, por ejemplo, los alumnos no entenderían el instituto como una cárcel, porque si ellos forman parte de la construcción de las normas...

$$
\text { (10_D_Sec_Pub_Des) }
$$

Se trata de una visión más «maleable» de lo que significa democracia, pues se considera un asunto a construir y el centro escolar, un espacio donde darle realidad. Por ello, tanto docentes como estudiantes destacan que la democracia requiere una comunicación y deliberación constante entre los miembros de la comunidad educativa. Las asambleas son un buen ejemplo de ello, pues permiten flexibilizar las normas y consensuar qué se considera democrático y qué no. El consenso se sitúa como mecanismo privilegiado para la toma de decisiones. Según los testimonios recogidos, el voto es un recurso secundario, como se refleja en este testimonio estudiantil:

Pues es que la democracia tiene una cosa básica que es la necesidad de una asamblea, donde la gente se siente a hablar. La democracia permite esa flexibilidad de conversación, esa capacidad de llegar a consenso y en el caso de que no se haga consenso, que se haga por votos. La democracia es eso, la organización política de un sistema en el que hay un espacio parlamentario donde se pueda hablar y donde se pueda debatir, que dé la flexibilidad a las leyes.

$$
\text { (11_E_Sec_Pri_Fav) }
$$

La comunicación también se considera esencial para examinar los problemas y retos de la vida escolar, pues la democracia es aquí mucho más que un asunto procedimental y normativo: se trata de valores comunes, de vincular a la gente y de mejorar la convivencia, por lo tanto, requiere también tratar los problemas de grupo:

Lo más esencial es la comunicación, tiene que haber una constante comunicación, un constante trámite de información, para ello también es necesario que la gente quiera interesarse en ello. Pero la posibilidad de información, además, de información abierta con transparencias, si hay un problema hay un problema.

(11_E_Sec_Pri_Fav)

Los resultados indican que la concepción participativa es aquella que supone una mayor igualdad entre todos los participantes. No hay roles radicalmente distintos ni disidencias notables sobre lo que docentes y estudiantes esperan de la democracia bajo esta concepción. El discurso es muy similar y apela a la posibilidad de participar directamente en las decisiones del centro, al compromiso y a la comunicación constante como medio de lograrlo.

\section{Discusión y conclusiones}

Los resultados de esta investigación sugieren tres concepciones diferentes sobre la democracia en el centro educativo: elistista, representativa y participativa. Estos hallazgos coinciden en buena parte con los principales resultados de la literatura internacional sobre el tema (Carr, 2008; Arensmeier, 2010; Carr et al., 2018). Sin embargo, invitan a matizar el espectro propuesto entre visiones representativas y participativas a partir de dos 
puntos principales. En primer lugar, la aparición de una visión de la democracia en la escuela muy restringida, que asociamos, al igual que Zyngier (2016), con visiones elitistas y tecnocráticas, donde la libre elección (especialmente de centro) tiene el papel central en la legitimación de los cargos de autoridad. En segundo lugar, la aparición de una visión crítica dentro de la concepción representativa, asumida por parte de un estudiantado que busca mayor injerencia y empoderamiento en los asuntos del centro y que matiza la asociación directa entre concepción representativa y democracia delgada.

La concepción elitista asume que el ejercicio de la democracia directa en la escuela no es posible ni deseable. La participación en los asuntos comunes es sumamente restringida: los jóvenes no tienen lugar en las decisiones del centro y las familias solo participan al elegir un centro educativo para sus hijos e hijas. El momento de libertad por excelencia, tal como reflejan los resultados, es el momento de elección de centro, de manera que la democracia queda asociada a la libre competencia en educación y la libre elección de centros. Al escoger el centro, las familias legitiman las decisiones que corresponden solamente a los técnicos (docentes, directivos, etc.).

Según esta concepción, cuando se trata de jóvenes, lo máximo que puede hacerse por la democracia es instruirlos en el tema. Esto sugiere que la propia democracia se vincula a un sentido técnico y a un conocimiento objetivo, pues se concibe como algo a ser enseñado aún cuando todavía no resulte adecuado ponerla en ejercicio. Esta visión se vincula a los discursos de la Nueva Gestión Pública que asocia la gestión con principios neoliberales y que promueve procesos de privatización de espacios públicos. Es necesario, por lo tanto, advertir acerca de lo restringido que resulta en relación con la participación comunitaria.

Los resultados de la investigación muestran que también en España está presente una concepción representativa de la democracia. Los hallazgos coinciden con los de Mathé (2016; 2019), pues sugieren una concepción de la democracia que reduce la participación al voto. También con los resultados del proyecto Democracia, alfabetización política y educación transformadora (Carr et al., 2018), pues en la mayoría de los casos se asocia con visiones más «delgadas» de la democracia. No obstante, revelan que existe un alumnado que, aún desde esta concepción, sostiene un discurso crítico y con ánimos de mayor empoderamiento. Este alumnado defiende la injerencia de todos los miembros de la comunidad educativa en la toma de decisiones importantes para el centro, incluso en asuntos tan fundamentales como la elección del director o directora. De este modo, la relación entre concepciones representativas de la democracia y visiones estrechas de la democracia queda en parte matizada.

Por último, la concepción participativa es, sin duda, aquella que engloba las visiones más «gruesas» de la democracia (Carr et al., 2018; Zyngier, 2016). La inclusión de toda la comunidad educativa en las decisiones del centro, la horizontalidad en sus relaciones y la igualdad entre todos y todas son esenciales para esta concepción. Además, los resultados indican que tanto docentes como estudiantes consideran el diálogo, la deliberación y el consenso como formas privilegiadas de realizar la democracia. Por último, ambos grupos entienden que, además de un procedimiento para tomar decisiones y el respeto a derechos básicos, la democracia es una construcción cotidiana que incluye también aspectos sustanciales y valores de convivencia. Más allá de sus hallazgos, esta investigación no estuvo exenta de dificultades. La mayor residió en las propias respuestas de las entrevistas, pues en muchos casos reflejaban ideas simples acerca de lo que significa la democracia en la escuela. A veces una misma respuesta planteaba ideas contradictorias entre sí, lo que dificultaba su categorización. Esto sugiere que docentes y estudiantes no tienen una concepción clara y desarrollada de lo que entienden por democracia en el centro educativo. Esta dificultad representa un hallazgo de la investigación que, sin ser buscado, resulta fundamental. Se trata de comprender que la debilidad para avanzar en una educación más democrática no está únicamente en concepciones que restringen la participación, sino en que no resulta un asunto lo suficientemente cercano (aunque sea discursivamente) como para que los agentes asuman, cuestionen y elaboren ideas propias sobre el fenómeno.

Entendiendo que la escuela tiene una responsabilidad directa sobre el modelo de sociedad que se pretende construir, es imperante promover la democracia como un asunto cercano, de ejercicio y reflexión. El camino es trabajar por el desarrollo de concepciones participativas de la democracia que modifiquen y enriquezcan las posturas más restringidas. Solo una concepción que entienda la democracia como un proceso en el que todos y todas tienen la misma capacidad de intervenir puede fomentar la agencia y el pensamiento crítico de las personas y, en última instancia, contribuir a la transformación hacia sociedades más justas y equitativas.

\section{Apoyos}

Este artículo ha sido desarrollado en el marco del proyecto financiado por el Plan Estatal I+D+i del Gobierno de España «La democracia en las escuelas como fundamento de una educación para la Justicia Social» (Ref. EDU2017-82688-P). 


\section{Referencias}

Åkerlind, G. S. (2008). A phenomenographic approach to developing academics' understanding of the nature of teaching and learning. Teaching in Higher Education, 13(6), 633-644. https://doi.org/10.1080/ 13562510802452350

Ángela Saiz-Linares, Rodríguez-Hoyos, C., y Susinos-Rada, T. (2019). 'I think we are still very directive': Teachers' discourses on democratic student participation. British Educational Research Journal, 45(1), 83-98. https://doi.org/10.1002/berj.3488

Arensmeier, C. (2010). The democratic common sense. Young Swedes' understanding of democracy - theoretical features and educational incentives. Young, 18(2), 197-222. https://doi.org/10.1177/ 110330881001800205

Barber, B. (1984). Strong democracy: participatory politics for a new age. University of California Press.

Barber, B. (1989). Public talk and civic action: education for participation in a strong democracy. Social Education, 53(6), 355-370. Retrieved from https://bit.ly/3psaPeR

Bengtsson, A.., y Christensen, H. (2016). Ideals and actions: Do citizens' patterns of political participation correspond to their conceptions of democracy? Government and Opposition, 51(2), 234-260. https://doi.org/ 10.1017 /gov.2014.29

Biseth, H. (2011). Citizenship education in Scandinavian multicultural schools: A comparative study of students and teachers perceptions. Citizenship Teaching \& Learning, 7(1), 71-88. https://doi.org/10.1386/ctl.7.1.71_1 Carr, P. R. (2008). Educadores y educación para la democracia: Trascendiendo una democracia «delgada». Revista Interamericana de Educación para la Democracia, 1, 146-166. Retrieved from https://bit.ly/3iSSm8N Carr, P. R., y Thésée, G. (2017). Seeking democracy inside, and outside, of education: re-conceptualizing perceptions and experiences related to democracy and education. Democracy \& Education, 25(2). Retrieved from http://bit.ly/3ppZBr6

Carr, P. R., Thésée, G., Zyngier, D., y Porfilio, B. J. (2018). Democracy, political literacy and transformative education (Informe final). Universidad de Québec. Retrieved from https://bit.ly/3ota4kl

Ceka, B., y Magalhães, P. C. (2020). Do the Rich and the Poor Have Different Conceptions of Democracy? Socioeconomic Status, Inequality, and the Political Status Quo. Comparative Politics, 52(3), 383-412. https:// doi.org/10.5129/001041520×15670823829196

Cohen, A. (2016a). Between teachers' perceptions and civic conceptions: lessons from three Israeli civics teachers. Journal of Curriculum Studies, 49(4), 542-560. https://doi.org/10.1080/00220272.2016.1263896

Cohen, A. (2016b). Navigating competing conceptions of civic education: lessons from three Israeli civics classrooms. Oxford Review of Education, 42(4), 391-407. https://doi.org/10.1080/03054985.2016.1194262

Durden, G. (2018). Accounting for the context in phenomenography-variation theory: Evidence of English graduates' conceptions of price. International Journal of Educational Research, 87, 12-21. https://doi.org/ 10.1016/j.ijer.2017.11.005

Edling, S., , y and, J. L. (2018). Student teachers' task perceptions of democracy in their future profession a critical discourse analysis of students' course texts. Australian Journal of Teacher Education, 43(7), 82-97. https://doi.org/10.14221/ajte.2018v43n7.5

Ferreras-Listán, M., Pineda-Alfonso, J. A., y Guichot-Muñoz, E. (2018). Teachers of secondary school as Democracy Coaches: Study of their conceptions during their initial formation. SHS Web of Conferences, 48, 01003-01003. https://doi.org/10.1051/shsconf/20184801003

Gandin, L. A., y Apple, M. W. (2002). Thin versus thick democracy in education: Porto alegre and the creation of alternatives to neo-liberalism. International Studies in Sociology of Education, 12(2), 99-116. https://doi.org/ $10.1080 / 09620210200200085$

Ginocchio, L., Frisancho, S., y Rosa, M. I. L. (2015). Concepciones y creencias docentes sobre la democracia en el colegio. Revista Peruana de Investigación Educativa, 7(7), 5-29. https://doi.org/10.34236/rpie.v7i7.48 González-Ugalde, C. (2014). Investigación fenomenográfica. Magis. Revista Internacional de Investigación en Educación, 7(14), 141-141. https://doi.org/10.11144/javeriana.m7-14.infe

Hahn, C. L. (2015). Teachers' perceptions of education for democratic citizenship in schools with transnational youth: a comparative study in the UK and Denmark. Research in Comparative and International Education, 10(1), 95-119. https://doi.org/10.1177/1745499914567821

Hung, Y.-H., y Perez, N. (2020). Exploration of immigrant in-service and pre-service teachers' conception and practice of citizenship in the United States. Citizenship Teaching \& Learning, 15(1), 101-116. https://doi.org/ 10.1386/ctl_00022_1

Kus, Z., , y Çetin, T. (2014). Perceptions of Democracy of Primary School Students. Educational Sciences: Theory \& Practice, 14(2), 786-790. https://doi.org/10.12738/estp.2014.2.1493

Landwehr, C., y Steiner, N. D. (2017). Where Democrats Disagree: Citizens' Normative Conceptions of Democracy. Political Studies, 65(4), 786-804. https://doi.org/10.1177/0032321717715398

Long, J. (2018). Educational administrators' perspectives of democracy and citizenship education: interviews 
with educational leaders. Journal of Contemporary Issues in Education, 13(2), 2-20. https://doi.org/10.20355/ jcie29349

Marton, F. (1981). Phenomenography-describing conceptions of the world around us. Instructional Science, 10, 177-200. Retrieved from https://bit.ly/2MyA116

Marton, F. (1986). Phenomenography: A research approach to investigating different understandings of reality. Journal of Thought, 21(3), 28-49. Retrieved from https://bit.ly/3j6e3Cx

Marton, F., y Booth, S. (1997). Learning and awareness. Mahwah: Lawrence Earlbaum.

Mathé, N. E. H. (2016). Students' Understanding of the Concept of Democracy and Implications for Teacher Education in Social Studies. Acta Didactica Norge, 10(2), 271-289. https://doi.org/10.5617/adno.2437

Mathé, N. E. H. (2019). PhD revisited: Students' Perceptions of Democracy, Politics, and Citizenship Preparation and Implications for Social Studies Education. Nordic Journal of Comparative and International Education (NJCIE), 3(1), 105-115. https://doi.org/10.7577/njcie.3355

Michels, R. (2008). Los partidos políticos: un estudio sociológico de las tendencias oligárquicas de la democracia moderna. Amorrortu.

Munck, G. L. (2016). What is democracy? A reconceptualization of the quality of democracy. Democratization, 23(1), 1-26. https://doi.org/10.1080/13510347.2014.918104

Murillo, F. J., y Hidalgo, N. (2018). Las concepciones sobre una evaluación justa de los estudiantes. Un estudio fenomenográfico desde la perspectiva de los docentes. Revista Complutense de Educación, 29(4), 995-1010. https://doi.org/10.5209/rced.54405

Orgill, M. (2012). Phenomenography. In N. M. Seed (Ed.), Encyclopedia of the Sciences of Learning (p. 26082611). Springer.

Quaranta, M. (2020). What makes up democracy? Meanings of democracy and their correlates among adolescents in 38 countries. Acta Politica, 55(4), 515-537. https://doi.org/10.1057/s41269-019-00129-4

Saada, N. (2020). Perceptions of democracy among Islamic education teachers in Israeli Arab high schools.

The Journal of Social Studies Research, 44(3), 271-280. https://doi.org/10.1016/j.jssr.2020.05.003

Schumpeter, J. A. (1988). Capitalismo, socialismo y democracia. Orbis.

Sim, J. B.-Y., Chua, S., y Krishnasamy, M. (2017). "Riding the citizenship wagon": Citizenship conceptions of social studies teachers in Singapore. Teaching and Teacher Education, 63, 92-102. https://doi.org/10.1016/ j.tate. 2016.12 .002

Sin, S. (2010). Considerations of quality in phenomenographic research. International Journal of Qualitative Methods, 9(4), 305-319. https://doi.org/10.1177/160940691000900401

Sjöström, B., y Dahlgren, L. O. (2002). Applying phenomenography in nursing research. Journal of Advanced Nursing, 40(3), 339-345. Retrieved from https://bit.ly/3cjcYWz

Vidal, R., Raga, L., y Martín, R. (2019). Percepciones sobre la escuela democrática en Argentina y España. Educação e Pesquisa, 45. Retrieved from http://dx.doi.org/10.1590/S1678-4634201945188681 http://doi.org/ $10.1590 /$ S1678-4634201945188681

Westheimer, J., y Kahne, J. (2004). What Kind of Citizen? The Politics of Educating for Democracy. American Educational Research Journal, 41(2), 237-269. https://doi.org/10.3102/00028312041002237

Zyngier, D. (2011). Rethinking the Thinking on Democracy in Education: What Are Educators Thinking (and Doing) About Democracy? Education Sciences, 2(1), 1-21. https://doi.org/10.3390/educ2010001

Zyngier, D. (2013). Democracy Will Not Fall From The Sky1. The Global Doing Democracy Research Project: Seeking to Understand the Perspectives, Experiences and Perceptions of Teachers in Relation to Democracy in Education. World Studies in Education, 14(2), 5-23. https://doi.org/10.7459/wse/14.2.02

Zyngier, D. (2016). What future teachers believe about democracy and why it is important. Teachers and Teaching, 22(7), 782-804. https://doi.org/10.1080/13540602.2016.1185817

Zyngier, D., Traverso, M. D., y Murriello, A. (2015). A comparative study of teacher education students' perceptions of democracy in two neo-liberal societies: Argentina and Australia. Research in Comparative and International Education, 10(2), 275-299. https://doi.org/10.1177/1745499915571709 